\title{
Un'azione edu-formativa «smart» come via italiana alla città intelligente: mediateca e cultura digitale
}

\section{Luca Luciani}

Università degli Studi di Padova, Dipartimento di Filosofia, Sociologia, Pedagogia, Psicologia Applicata

doi: 10.7358/ecps-2014-010-luci

luca.luciani@unipd.it

luca.luciani.ll@gmail.com

\section{A SMART EDUCATIONAL ACTION AS THE ITALIAN PAT TO THE SMART CITY: THE MULTIMEDIA PUBLIC LIBRARY AND DIGITAL CULTURE}

\section{Abstract}

Although the term "smart city" is increasingly used today, it is open to different interpretations and uses. Much like an increasing fashion that is easy to adopt, the smart city is being increasingly proclaimed, proposed and considered a goal by policymakers. Many administrative actions and applications are called "smart", but what is a "smart city" exactly? When can a city actually be considered smart? What is the relationship between the "smartness" of its technological-organizational systems of social life, of its environment or territory and of its citizens' intelligence? Considering the development and evolution of the modern city, is there a relationship between intelligence and creativity? Through the answers to these questions, the result of a logical-consequential theoretical analysis, and with regard to the critical acquisition and spreading of a digital culture, this contribution aims to put forward and support the value linked to creating a widespread network of multimedia public libraries, proposed as a cornerstone for actually achieving a smart city. This action must not be delayed in Italy seeing as it significantly lags behind other European and more economically advanced countries in this regard. Italy is sorely lacking in this kind of library with its far-reaching cultural benefits that can potentially enhance human socio-creative development of «digital intelligence».

Keywords: Creativity, Digital literacy, Media education, Multimedia public library, Smart city. 


\section{IL PROBLEMA EDUCATIVO NELLE AZIONI PER LA CITTÀ INTELLIGENTE}

A ben scandagliare le maglie strutturali delle indicazioni generali, e più ancora dei diversi bandi progettuali che vengono proposti in relazione allo sviluppo delle smart cities, emerge con chiarezza come uno dei temi fondanti di questo auspicabile e progressivamente possibile divenire degli agglomerati abitativi, economici, e di vita socio-culturale, che denominiamo città, sia quello della «città educante». Spesso però negli aspetti realizzativi dei vari progetti si osserva che lo sviluppo di questa nuova potenziale dimensione educativa, anziché concretizzarsi come uno spazio agito attraverso azioni/ processi di sperimentazione e ricerca, al fine di raggiungere un miglioramento ed un adeguamento formativo della persona, risulta invece soltanto come uno dei possibili ambienti della città dove proporre nuovi elementi organizzativi digitali e non (spostamento ottimizzato di alunni e genitori, iscrizioni e vari servizi erogati online attraverso computer o smartphonel tablet, ecc.), e azioni di risparmio energetico (tetti fotovoltaici, ristrutturazione delle sedi scolastiche con nuovi isolamenti, utilizzo di nuovi combustibili, ecc.). In definitiva, si tratta di innescare una maggiore funzionalizzazione ecologica per un miglioramento complessivo della dimensione dinamico-sociale della città.

L'analisi delle proposte di smart education che ad oggi provengono dagli enti, dalle amministrazioni pubbliche, dalle agenzie, dalle imprese e dalle stesse università dimostrano di non volersi porre compiutamente in correlazione con le reiterate richieste ed indicazioni europee ed internazionali (Commissione Europea, UNESCO) relative alla necessità di un sistema eduformativo che permetta alle persone di svilupparsi anche al fine di poter svolgere in modo completo ed efficace il proprio ruolo di cittadinanza attiva. Si rinuncia così ancora una volta a dotare l'insieme del sistema sociale di quella complessa, articolata e significativamente strutturata «infrastruttura del sapere», con la quale formare oggi tutte le diverse generazioni di persone, con una particolare attenzione alle nuove generazioni e a quei cittadini, che nella dimensione competitiva socio-economica globale e glocale, soprattutto in un periodo di crisi di sistema come quello che stiamo vivendo, abbisognerebbero di una reale e validata formazione continua al fine di provare a reintrepretare il più possibile positivamente $\mathrm{i}$ costanti cambiamenti in essere.

Questo stato di cose è possibile rilevarlo anche dagli esiti del recente bando del MIUR Avviso per la presentazione di idee progettuali per smart cities and communities and social innovation (MIUR, 2012a). Il bando presentava tra i diversi ambiti anche quello specifico dell'educazione introducendolo così: 
[...] sostenere la progettazione di devices innovativi destinati agli studenti, in grado di supportare sia la lettura del libro elettronico, con schermi e risoluzioni idonee, sia l'accesso e l'utilizzo, con architetture aperte ai principali sistemi operativi, di contenuti digitali multimediali accessibili in rete; Learning Management System (LMS) in grado di supportare tutte le funzioni di gestione necessarie alla personalizzazione dei percorsi di apprendimento, in termini di flessibilità degli orari e affiancamento alle attività in presenza, articolazione dinamica dei gruppi e strumenti per la gestione degli studenti; sistemi di Content Management System (CMS), integrabili in ambienti LMS, rivolti agli insegnanti per lo sviluppo di contenuti digitali multimediali.

Già da questa presentazione si evince come la problematica educativa nella città intelligente, almeno quella attualmente proposta in Italia, tenda a risolversi con parziali proposte di innovazione tecnica più che tecnologica, laddove per tecnologia educativa si voglia intendere anche la messa a punto e la calibrazione di particolari percorsi/processi didattici (Galliani, 2000). Innanzitutto tale ambito edu-formativo avrebbe potuto essere sviluppato in modo trasversale anche in altre aree proposte dal bando e non essere soltanto specificatamente incardinato all'ambito educativo della scuola: per esempio in quello dell' «invecchiamento della società», soprattutto in funzione della formazione continua e flessibile, o in quello delle "tecnologie welfare e inclusione», soprattutto in relazione al cultural and digital divide sociale, o ancora, in quello della «salute», a livello dei processi educativi legati alla prevenzione (per esempio l'alimentazione nelle generazioni più giovani), o nell'ambito del cultural heritage, per generare in modo più esteso e approfondito possibile nella popolazione il senso del valore socio-culturale ed economico del nostro patrimonio culturale inteso anche nel senso delle varie espressioni creative contemporanee. Da una sua attenta disamina si evince invece che solo un progetto, tra i diciassette approvati, si è orientato verso l'educazione. Si tratta del progetto dal titolo Smart education - EDOC@WORK 3.0 (MIUR, 2012b).

Una decisa conferma in tal senso ci viene da Carlo Giovannella (2012 e 2013), che da tempo lavora all'idea di smart city come nuovo approccio per lo sviluppo della qualità della vita attraverso una prospettiva anche necessariamente educativa: smart cities learning. Si tratta di un approccio che tiene in forte considerazione gli aspetti umani costituiti da motivazioni, aspettative, desideri, bisogni, stili di vita personali e che nelle azioni progettate e realizzate per le città in prospettiva smart finiscono inevitabilmente per correlarsi ai processi di ottimizzazione tecnica urbana ormai quasi sempre sospinti dalle nuove Tecnologie dell'Informazione e della Comunicazione (TIC). Intervistato da Roberta Gatti (2012) Giovannella indica in modo chiaro come «a parole tutti sembrano comprendere l'importanza dell'educazione [...]. Nella pratica 
però, lo spazio riservato nei bandi a questo tema è minimo e la costruenda 'palestra italiana' traguardata ad Horizon 2020 sembra schiacciarsi su modelli di smart city in cui i cittadini sono poco più che degli utenti necessari al funzionamento del sistema». Riferendosi specificatamente alle istituzioni scolastiche e formative formali rileva come "in tutti i report e modelli di smart city l'educazione è vista come un fattore abilitante in grado di incidere sulla qualità della vita esclusivamente in termini infrastrutturali e funzionali: densità di scuole, erogazione di contenuti e servizi tramite infrastrutture a banda larga, capacità di produrre le skill necessarie al funzionamento del sistema». In questo modo è chiaro che la riflessione sulle modalità di apprendimento $\mathrm{e}$ la centralità della persona, che è prospettiva trasversale alle altre tematiche di riferimento per le smart cities, avrà scarse o nulle possibilità di concretizzarsi e di apportare il suo necessario e importante potenziale trasformativo al processo realizzativo della città intelligente.

Sebbene il concetto di territorio non sia perfettamente sovrapponibile a quello di città, una città con le sue espansioni/correlazioni suburbane può essere comunque considerata anche come un «insieme territoriale» e in modo particolare un insieme comunicativo-relazionale tra le persone (Galliani, 1983). Se in questo contesto il fattore trasversale, che dovrebbe essere comune a tutte le azioni che si promuovono per sostenere la trasformazione delle città in smart cities, sono le persone, allora il loro sviluppo formativo diventa prioritario e centrale. Infatti, possono essere soltanto gli smart citizens, ovvero, individui che si sentiranno «intelligenti» perché compartecipi dei vari progetti, e non solo per avere appreso/assunto delle tecniche di ottimizzazione funzionale, ma anche per essere diventati parte effettiva dell'innovazione tecnologica e del cambiamento operativo introdotti, che saranno in grado di valorizzare al meglio la qualità delle risultanze evolutive che vengono a determinarsi. E questo può arrivare ad effettivo compimento solo assumendo da interpreti attivi, nella propria completa dimensione umana, costituita da emozioni, ideali, cultura, che implicano motivazioni, aspettative, desideri, bisogni, e nei propri stili di vita, oltre alla dimensione funzionale, anche il portato di senso e di utilizzo consapevole dell'innovazione tecnologica. Peraltro anche lo sguardo economicista conferma come «il ruolo economico del territorio [sia] sempre più legato non tanto alle convenienze localizzative classiche (distanza, posizione, dotazioni, fertilità, ecc.) quanto al circuito cognitivo che, in funzione della storia e dell'organizzazione sociale, si è sedimentato nei luoghi, rendendoli diversi l'uno dall'altro» (Rullani, 2006, p. 48). Il fulcro, allora, nei processi dell'agire territoriale al fine di raggiungere una reale e stabile efficienza ed efficacia delle azioni trasformative, in grado anche di innalzare, modificare, o stabilizzare, i livelli economici dei territori, che possiamo riassumere nell' "essere competitivi», diventano chiaramente 
l'educazione e la formazione nelle loro dimensioni formali, non formali e informali.

Anche l'urbanista inter e multi-disciplinare Charles Landry inserisce in qualche modo al primo posto delle azioni per fare la città (city making) l'educazione e la formazione. In opposizione alle denominazioni di «città buona», "città della conoscenza», "città intelligente» propone quella di "città che impara»: "[...] una città creativa, che apprende in continuazione, è una città intelligente che riflette su se stessa, impara dai propri errori ed è strategica: perché la città è un campo scuola» (Landry, 2009, p. 361). Gli spazi e le risorse per l'apprendimento sono ovunque da quelle formali come le scuole, ovviamente, a quelle non formali come il variegato mondo dell'associazionismo e delle istituzioni/strutture non scolastiche, oltre a tutte quelle informali più impensabili: ambienti della strada, carceri, centri commerciali, nuove tecnologie della comunicazione, in modo particolare i social network, e in realtà ogni ambiente in cui si riesca ad individuare una possibile e significativa azione edu-comunicativa. La "città che impara» è un ambito territoriale e un ambiente che valorizza, e quindi stimola, promuove e sostiene le persone nel loro costante sviluppo e miglioramento conoscitivo. In questo senso è uno spazio agito in grado di comprendersi sempre meglio e di riflettere su questo processo di conoscenza diventando così anche una «città riflessiva» e sviluppando quel necessario grado di resilienza creativa che può permetterle di svilupparsi con successo in una dimensione socio-economica in veloce e costante trasformazione. A questo proposito sappiamo tra l'altro da Dewey (2012) quanto più performante e quanto più profondamente assimilato sia un apprendimento in cui venga incentivato il livello riflessivo dell'esperienza edu-formativa. Quando si scoprono le connesioni dettagliate delle attività e di ciò che comportano come conseguenze, il pensiero implicito nell'esperienza acquisisce un rilievo qualitativo, quantitativo e un valore molto diverso. Questo fa sì che cambi la qualità dell'esperienza e «[...] il cambiamento [risultante] è così significativo che possiamo chiamare questo tipo di esperienza riflessivo, cioè riflessivo eminentemente. Il coltivare deliberatamente questa fase di pensiero costituisce il pensare come esperienza a sé. Pensare è, in altre parole, il tentativo intenzionale di scoprire connessioni specifiche fra qualche cosa che facciamo e le conseguenze che ne risultano, in modo che le due cose diventino continue» (Dewey, 2012, p. 158). Allora, per tornare al problema educativo nella "città intelligente», diventa conseguente come «la sfida che ha di fronte a sé chi decide quali politiche adottare è quella di promuovere le condizioni in cui possa svilupparsi una città o una comunità che voglia imparare. Si tratta di qualcosa che va ben oltre al classico studio scolastico, perché si tratta invece di creare un luogo che sia totalmente impregnato dell'idea di apprendimento» (Landry, 2009, p. 362). 
In questo modo la smart city si configura come una "comunità educante» ed è proprio questa attenzione e azione trasversale che è in grado di dare maggiore efficienza ed efficacia ai processi tecnico-strutturali di ottimizzazione del «consumo di risorse e di fluidificazione dei flussi che l'attraversano (cose, persone, dati)» (Giovannella in Gatti, 2012). L'educazione e la formazione quindi, devono diventare concetti cardine per lo sviluppo innanzitutto di una "comunità intelligente», creando capitale sociale, competenze cognitive e relazionali, partecipazione e senso democratico. Le istituzioni educativoformative di ogni genere e grado (scuole, università), come anche tutte le attività della formazione permanente che si collocano nell'ambito non formale ed informale (istituzioni culturali pubbliche e private, associazioni, enti del territorio, sistemi di comunicazione sociale online e movimenti/aggregazioni/gruppi/ spontanei di interesse e comunicazione sociale, ecc.) sono allora di fatto attori fondamentali nel complesso delle possibili azioni per la «città intelligente».

\section{DALLA «CITTÀ INTELLIGENTE» ALLA "CITTÀ CREATIVA»: ANDATA E RITORNO}

Abbiamo visto come in relazione alle azioni progettate con l'obiettivo della "città intelligente» la quasi totalità di queste riguardino dei «meccanismi automatizzati» di tipo tecnologico come anche di tipo ambientale. Una nuova infrastrutturazione urbana digitale e non, anche in diversi casi ecologica, ma che non prevede nel suo processo di costruzione/implementazione la diretta presenza partecipata delle persone-cittadini. La città viene fornita di più attuali, più efficienti ed efficaci dotazioni funzionali nei confronti delle quali le persone-cittadini vengono soltanto invitate a fruirne disegnando per loro un ruolo di fatto passivo e di mero adeguamento funzionale. Si sviluppa così una sorta di «città robotica» dove alle persone-cittadini viene chiesto di aggiornarsi e di adattarsi per poterla adottare fruendola.

Proviamo a fare un semplice esempio. Immaginiamo un'amministrazione cittadina che doti la città di un sistema di acquisto online dei biglietti per il trasporto pubblico funzionante anche dal proprio telefono mobile collegato alla rete internet. Si tratta certamente di un servizio innovativo e vantaggioso in termini funzionali di movimento e spazio. Probabilmente, la comodità e la possibilità di acquisto anche a qualsiasi fermata o sul mezzo nei momenti concitati in cui si sale "al volo», permetterà di stimolare una maggiore propensione al corretto acquisto del biglietto in qualsiasi situazione d'uso del mezzo pubblico oltre ad un possibile incremento dell'utilizzazione stessa dei 
mezzi pubblici: un valido servizio rivolto a chi ha le necessarie competenze tecnologiche e possiede le relative tecnologie. Di fatto però questa azione non incide in alcun modo sulle contemporanee disuguaglianze e le conseguenti riduzioni di opportunità di una comunità nel suo insieme derivanti dal digital divide generazionale (over 45) e a volte anche intergenerazionale.

È questa la «città intelligente» che ci aspetta? Ė questa la «città intelligente» che permette di elaborare una significativa resilienza collettiva in grado di rispondere alle continue sfide competitive poste da una società e una economia globale in continua evoluzione e cambiamento? È questa la città intelligente che ci permetterà di sviluppare quella dimensione comunitaria in grado di favorire e valorizzare la cittadinanza attiva e critica, la partecipazione ai processi evolutivi, la sempre più necessaria formazione continua, che tra l'altro sono sollecitate ufficialmente dalle istituzioni europee e purtroppo spesso solo di riflesso, anche da quelle nazionali? (Commissione Europea, 1995 e 2010; Consiglio d'Europa, 2008). Fino a che punto possiamo considerare intelligente questa "automazione» nell'ottimizzazione del consumo di risorse e nella fluidificazione dei flussi di cose, persone, dati, e quindi nell'adeguamento tecnologico dei servizi in modo particolare pubblici e in riferimento all'utilizzo delle TIC? Crediamo che le risposte a queste domande dipendano essenzialmente dal paradigma utilizzato nella progettazione delle azioni per la smart city: se al centro dei processi trasformativi è posto l'essere umano nella sua interezza, diversità e complessità, e quindi ci si rivolge ad un attore compartecipe, consapevole, pro-attivo, oppure, al contrario, se le persone-cittadini vengono considerate come dei semplici destinatari esecutori e/o fruitori, riservando loro un ruolo di mero adeguamento più o meno attivo.

Una risposta a questo possibile cambio di paradigma da adottare nelle prassi ideative e realizzative per la "città intelligente» ce la fornisce Charles Landry (1998, 2009, 2012 e 2013) rivalutando il senso della creatività e inserendola come dimensione costitutiva del «fare città». Non si tratta più di una creatività esclusivamente correlata agli ambiti più facilmente riconosciuti per la loro inventiva come quello dell'intrattenimento, del mondo della moda, delle celebrità mediatiche. La creatività non è più solo una loro prerogativa, come peraltro non lo è mai effettivamente stata, ma riguarda ogni sfera dell'agire umano. Anche attività che siamo indotti a pensare dai cliché della comunicazione mediale come escluse dal poter essere creative, il lavoro sociale per esempio, in realtà non lo sono. Eroine ed eroi creativi, e Landry li introduce proprio con queste parole, si possono trovare tra gli imprenditori sociali, tra gli scienziati, tra le persone che si occupano di affari, tra i pubblici amministratori, tra gli artigiani, ecc. In questo modo la rivalutazione della creatività impone quindi di riconsiderare il suo ambito di azione e le sue pos- 
sibili applicazioni. La creatività inoltre dovrebbe anche essere investita di valori etici. In questo modo i suoi campi di applicazione dovrebbero riguardare anche le sfide alla povertà, l'attenzione all'ambiente, l'innovazione politica e sociale. "In che modo può essere rinnovata la democrazia, come possono cambiare i nostri comportamenti, come si possono riallineare le gerarchie, come si possono riformare le carceri e il sistema delle pene, come potrebbe essere l'assistenza sociale, come possono sentirsi impegnati i giovani, come può essere innescata la creatività collettiva e di massa: sono esattamente queste le aree che richiedono maggiormente il nostro sforzo intellettuale e che non possono rinunciare ad approcci creativi» (Landry, 2009, p. 313).

In uno studio del 1998 relativo a venti progetti creativi per la città di Helsinki proposti nei più disparati campi di azione, questo urbanista interdisciplinare ha potuto constatare come gli ideatori e le persone chiave della loro strutturazione possedessero caratteristiche individuali in discipline tra loro completamente differenti. Le caratteristiche messe in evidenza sono: mentalità aperta e incline alla scoperta, capacità di focalizzare i problemi e le situazioni, un approccio mentale anticonvenzionale e flessibile. Il milieu creativo necessario allo sviluppo della "città intelligente» allora sarà costituito dalla sfida "nel valutare e mettere in relazione diverse forme di creatività nella sfera ambientale, politica, economica, sociale e culturale» (ibid.), tenendo sempre presente che "fondamentalmente la creatività è un viaggio, non una meta, un processo, non uno stato» (ivi, p. 314).

Lo scenario in cui l'urbanista multidisciplinare Richard Florida ambienta l'ascesa e le potenzialità di sviluppo socio-economico derivanti da quella che ha definito "classe creativa» è proprio la città. Relativizzando l'idea che i vantaggi geografici siano stati completamente annullati dal consistente sviluppo della comunicazione e dall'ubiquità comunicativa delle reti e della rete internet in particolare, Florida elabora la "teoria del capitale creativo». La classe creativa delineata da Florida, suddivisa, in base alla tipologia di processo creativo che caratterizza i diversi lavori, in "nucleo supercreativo» (scienziati, ingegneri, docenti universitari, poeti, romanzieri, programmatori di software, artisti, registi, attori, stilisti, architetti, scrittori di testi non narrativi, dirigenti editoriali, figure del mondo culturale, ricercatori, analisti, opinionisti, ecc.), e "creativi di professione" (professioni ad alto impiego di conoscenze: dell'ambito legale, sanitario, di quello finanziario, della direzione aziendale, ecc.), si disloca e si accentra preferendo «i luoghi diversi, tolleranti e aperti alle nuove idee» (Florida, 2003, p. 295): è questa "concentrazione» di classe creativa che contribuisce a favorire lo sviluppo socioeconomico di una regione o di una città. La "qualità del luogo» è in grado di attrarre la classe creativa e tale qualità è indicata dalle categorie indicate come le «tre T»: la tecnologia (presenza di imprese high-tech e/o registrazione 
di un elevato numero di brevetti, per esempio), il talento (presenza di persone con un elevato grado di istruzione), la tolleranza (un numero significativo di stranieri inseriti nel sistema sociale e di omosessuali (per estensione, di persone appartenenti alla dimensione LGBT). Si tratta di una visione più "elitaria» e "aristocratica», peraltro risultato di studi scientifici che hanno comportato l'analisi dei dati sociali e produttivi dei maggiori distretti americani passati poi attraverso le maglie degli indicatori inseriti nel creativity index, anche in buona parte in linea con il Manifesto per la creatività e l'innovazione in Europa (Commissione Europea, 2009), rispetto al concetto di creatività proposto per la resilienza e lo sviluppo positivo delle città da parte di Landry, più distribuito, più partecipato, più aperto, in fondo più democratico, e a cui personalmente ci sentiamo di aderire maggiormente. Questa proposta concettuale comunque, come quella precedente e quella promulgata dall'UNESCO, hanno il merito di considerare e stimolare la problematica delle decisioni pubbliche dirette sia ad incoraggiare la creatività come anche a porla come elemento catalizzatore e centrale dei processi correlati alle azioni per la «città intelligente».

$\mathrm{La}$ "città che impara» ad essere "creativamente intelligente» attraverso la trasformazione e la crescita partecipata prima di tutto dei suoi stessi cittadini trova anche delle validazioni e dei supporti teorico-metodologici nell'ambito della pedagogia e della psico-pedagogia. In modo particolare in quel fecondo solco pragmatista "trasformativo» e "progressivo» che a partire da Dewey arriva in linea di discendenza sostanzialmente diretta a Gardner. Questo è un ambito della pedagogia e della psico-pedagogia dove la creatività nell'esperienza conoscitiva delle persone è stata nel tempo significativamente correlata alla dimensione pubblica, sociale e socio-economica delle organizzazioni. Se Dewey pone in relazione diretta l'educazione e la democrazia attraverso la stimolazione delle capacità espressive, creative e di conoscenza (1917 e 2012), Gardner giunge ad individuare il potenziale creativo non nelle sole capacità talentuose dell'individuo ma anche in correlazione ai diversi possibili campi di conoscenza in cui può decidere di applicarsi e all'influenza dello specifico contesto sociale di cui è contornato (1991), arrivando successivamente a far comprendere il valore sociale ed economico strategico della creatività alle organizzazioni produttive e alla sfera sociale nei rapporti umani (2007). Si tratta di ulteriori importanti stimoli teorico-metodologici che vanno a supportare l'idea di una "città intelligente» perché creativa ricordando e sottolineando in modo particolare con Gardner che «se la creatività non penetra nel DNA di una organizzazione[, come per il nostro discorso di una città,] è improbabile che venga trasmessa alla generazione successiva, [... e che] la creatività è sempre qualche cosa di casuale, e che quindi non può essere garantita ma solo coltivata e stimolata» (2007, pp. 98-99). 
La specifica relazione dinamica e positivamente attiva tra la creatività culturale e la città è riconosciuta e promulgata anche dall'UNESCO (Creative Cities Network). Il network delle città creative dell'UNESCO, al quale, a cadenza annuale, attraverso una determinata procedura e garantendo uno specifico processo di autovalutazione, varie città potrebbero provare a raggiungere gli standard necessari per farne parte nelle diverse categorie previste, propone come obiettivi qualificanti e strategici per lo sviluppo della città: la creatività come elemento essenziale per lo sviluppo economico e sociale locale; la creatività come sviluppo di nuove possibilità di cooperazione e partenariato; la creatività come terreno su cui sviluppare maggiori partnership pubblico/privato in grado di contribuire a sbloccare il potenziale imprenditoriale creativo e rivestire così un importante ruolo nella new economy; l'incremento/ miglioramento dell'industria culturale creativa della città in grado di portarla a svolgere un ruolo di gateway nei confronti dei mercati internazionali; la cultura come efficace motore di rinnovamento urbano; la creatività culturale e le sue industrie come strumento per il miglioramento della qualità della vita, per la costruzione del tessuto sociale, per lo sviluppo di equilibrio e tolleranza rispetto alla diversità culturale, per il rafforzamento del senso di comunità, per il contributo nella definizione di una identità condivisa.

La creatività allora diventa per la città una forma di capitale e si connota come «un'intraprendenza dalle molte sfaccettaure, è immaginazione che viene applicata attraverso l'uso di qualità come l'intelligenza, l'inventiva, imparando lungo il cammino. È dinamica e dipende dal contesto: ciò che in un certo periodo o in una certa situazione è creativo non necessariamente lo è in un altro" (Landry, 2009, p. 313). Soltanto una città partecipata con creatività possiederà quindi quel DNA resiliente capace di farla competere e migliorare costantemente sia nella dimensione globale quanto in quella locale. Così, allora, soltanto la città creativa potrà essere considerata una smart city, una città intelligente, anzi una città "creativamente intelligente».

\section{CONDENSATORI, STIMOLATORI, RACCORDATORI, ATTIVATORI DI CREATIVITÀ PER LA CITTÀ INTELLIGENTE}

Se quanto abbiamo evidenziato nei due precedenti paragrafi è una condizione corretta in relazione alla convenienza e alla modalità di strutturare le azioni e i relativi processi finalizzati al raggiungimento e alla costante evoluzione della "città intelligente», allora, oltre alle scuole/università nella loro dimensione educativa formale, c'è la necessità di individuare, preservare e rilanciare tutte quelle «istituzioni» culturali che nella loro dimensione educativa non formale 
e informale sono in grado di fungere da condensatori, stimolatori, raccordatori e attivatori della creatività sociale distribuita, diffusa, e collettiva, quanto individuale.

A questo proposito Landry (2009) condiziona l'esistenza di un milieu creativo in una città sia all'esistenza di un hardware infrastrutturale (edifici, strade, condutture, ecc.) quanto alla contemporanea esistenza di infrastrutture soft che comprendono la dimensione mentale, le attitudini del pensiero, il piano spirituale, le aspirazioni individuali e collettive: «[...] è l'infrastruttura intellettuale formale e informale» (ivi, p. 460). In modo particolare sembra essere la diversità culturale, la sua accettazione e la sua tendenzialmente positiva valutazione, che consente di rafforzare la città. Le città creative, infatti, sembrano avere bisogno di essere influenzate e positivamente contagiate dall'esterno con l'introduzione di nuove idee, prodotti e servizi, sfidando così gli equilibri in essere e arrivando a generare nuove situazioni combinatorie dove si relazionano, incontrandosi, la dimensione interna con quella esterna: «La sfida creativa, come è stato rimarcato, implica di passare dalla città multiculturale, dove riconosciamo almeno in teoria rispettiamo le nostre differenti culture, alla città interculturale. [... Questo nuovo spazio condiviso], verosimilmente, può portare a un maggior benessere e una maggiore prosperità» (ivi, p. 462). E per Landry sono proprio le istituzioni culturali, come per esempio le mediateche pubbliche, gli strumenti attivi in possesso della città per l'ancoraggio della "comunità in divenire» e per lo sviluppo della creatività. Attraverso la loro capacità di svelare e di costruire l'identità di una città nelle sue prospettive di passato, di presente e di futuro, sono in grado di produrre fiducia nelle persone che la abitano. Sono queste istituzioni che quindi permettono di posizionarci all'intersezione delle condizioni precedenti, di quelle presenti, e di quelle immaginate o progettate per il futuro. Così facendo, «diventano piattaforme per il dialogo, il discorso e il dibattito, rivelando le molteplici stratificazioni che costituiscono una società. In questi processi di creazione, di interrogazione e ancoraggio dell'identità, di immaginazione, reimmaginazione e scoperta, l'elemento catalizzatore è l'oggetto, o manufatto, idealmente reale $[\ldots$, , i loro valori sono impressi nel loro tessuto fisico tanto quanto nella loro programmazione» (ivi, p. 468).

Anche Florida (2003) pone l'accento sulla forza delle infrastrutture culturali che considera capaci di sostenere l'economia creativa. Così, grazie a tutte le possibili istituzioni culturali, tra cui le public libraries, oltre alla diffusa implementazione delle nuove tecnologie della comunicazione attraverso una rete capillare ed efficiente, le città e i territori possono diventare luoghi privilegiati per un costante e innovativo sviluppo socio-economico. Richard Florida scrive infatti che le città e i territori per favorire la nuova economia devono dotarsi di «un ambito sociale di sostegno, che sia aperto 
alla creatività in tutte le sue forme [...] e che fornisca l'ecosistema, l'habitat nel quale le forme più diverse di creatività mettono radici e fioriscono, [perché] incoraggiare stili di vita e manifestazioni culturali, quali ad esempio la musica d'avanguardia o una vivace comunità artistica, ha l'effetto di attrarre e stimolare anche chi crea nel campo degli affari e della tecnologia» (2003, p. 85). Tra gli esempi che porta relativi alle strutture in grado di creare l'ecosistema per la creatività c'è anche quello della public library. E lo fa ricordando e mettendo in rilievo l'importanza che riserva ad un tratto biografico della sua diretta esperienza personale: "[...] i sabati che passavo con mio padre [...] alla Newark Public Library, dove mi lasciava girare fra gli scaffali, e dove divoravo volumi e volumi sull'America urbana» (ivi, p. 366).

Il Sole 24 Ore, il quotidiano nazionale che maggiormente si occupa di economia e finanza in Italia, il 19 febbraio del 2012 presenta il Manifesto della Cultura sul supplemento domenicale dal titolo Niente cultura, niente sviluppo. Si tratta di un avvenimento editoriale che lancia una riuscita raccolta di adesioni a cui succedono l'ideazione e l'organizzazione di due edizioni degli «Stati Generali della Cultura» oltre ad un ampio, approfondito e costante dibattito nazionale. L'obiettivo dichiarato era ed è tutt'ora il rilancio economico del paese attraverso la cultura. Aderiscono nel tempo oltre a dibattere e intervenire su questa tematica vari ministri, lo stesso attuale Presidente della Repubblica, imprenditori, economisti, personalità del mondo della cultura. Alcuni passaggi salienti di questo Manifesto mettono in chiara relazione lo sviluppo economico con la cultura e la creatività. Infatti, in questo testo si legge che «i beni culturali e l'intera sfera della conoscenza devono tornare ad essere determinanti per il consolidamento di una sfera pubblica democratica, per la crescita reale e per la rinascita dell'occupazione» (Il Sole 24 Ore, 2012). Il discorso viene proposto in chiave strettamente economica: «Niente cultura, niente sviluppo» (ibid.). La cultura è qui intesa in modo ampio e implica educazione, istruzione, ricerca scientifica, conoscenza, mentre lo sviluppo non viene considerato nella sua dimensione esclusivamente economicista incentrata sull'aumento del Pil. Per il Manifesto è necessario porsi in «un'ottica di medio-lungo periodo in cui lo sviluppo passi obbligatoriamente per la valorizzazione dei saperi, delle culture, puntando in questo modo sulla capacità di guidare il cambiamento. La cultura e la ricerca innescano innovazione, e dunque creano occupazione, producono progresso e sviluppo» (ibid.). Lo spazio di azione privilegiato di queste linee guida viene individuato specificatamente nelle città: «[...] anche la crisi del nostro dopoguerra, a ben vedere, fu affrontata investendo in cultura. Le nostre città, durante quella stagione, sono state protagoniste della crescita, hanno costruito 'cittadini', e il valore sociale condiviso che ne è derivato ha creato una nuova cultura economica. [...] Se oggi quelle stesse città che sono state laboratori viventi sembrano 
traumatizzate da un senso di inadeguatezza nell'interpretare le nuove sfide, ciò va ascritto a precise responsabilità di governo e a politiche e pratiche decisionali sbagliate» (ibid.). Nel Manifesto la possibilità di sviluppare la necessaria creatività sociale e individuale per lo sviluppo futuro è fortemente ancorata a tutti i livelli educativi oltre ad essere trasversale a tutte le discipline con una necessaria particolare attenzione, che quasi allo stesso modo è stata evidenziata anche da Landry (2009) e che è anche in linea con il pensiero precursore di Dewey (2012), per lo studio dell'arte e della storia. A questo proposito il Manifesto precisa che "per studio dell'arte si intende l'acquisizione di pratiche creative e non solo lo studio della storia dell'arte» (Il Sole 24 Ore, 2012).

In relazione alle linee guida del Manifesto della Cultura e in riferimento alle proposte di Richard Florida, si innesta convenientemente e con precisione la riflessione dell'esperta, studiosa, e progettista di biblio-mediateche Antonella Agnoli (2009). Proprio a partire dalla considerazione della crisi italiana, le biblioteche di pubblica lettura vengono individuate come una parte necessaria della soluzione. In un paese in cui molteplici indicatori mostrano un tasso di ignoranza crescente e che rischia per questo di essere marginalizzato nella dimensione glocale dell'economia della conoscenza, la bibliomediateca come luogo di libertà e di creatività per ogni cittadino dovrebbe diventare parte di un progetto di rinascita di questo paese. La biblio-mediateca pubblica come territorio aperto a gruppi e associazioni, come centro di riflessione e di condivisione dei saperi, come nodo centrale di una rete con le altre istituzioni culturali, può effettivamente incidere sull'ecosistema culturale creando quell'ambiente necessario a far si che si possano attivare le più diverse forme di creatività. Per conseguire questo obiettivo «non si può che partire dalle città e avviare servizi che, nel lungo periodo, stimolino la lettura, la conoscenza della musica, del cinema, dell'arte. Questi servizi hanno senso soltanto se sono collegati fra loro, se collaborano, se formano una rete [...]: biblioteche rinnovate potrebbero [...] dare un impulso alla collaborazione fra istituzioni diverse, oltre che indirizzare il cittadino verso altre esperienze culturali sul territorio [...]» (Agnoli, 2009, pp. 63-64).

Sullo sfondo dello scenario della progressiva trasformazione, avvenuta in modo particolare in Francia a partire dagli anni '70/'80, delle biblioteche in mediateche a causa della necessaria integrazione di molteplici media con i loro differenti supporti tecnologici e del metamedium computer collegato alla rete Internet, Corbineau (2005) rileva il ruolo chiave di questi istituti culturali in relazione allo sviluppo delle politiche e delle conseguenti azioni per conseguire l'intelligenza territoriale in termini di sviluppo duraturo e flessibile. Si tratta di un ruolo ancora sottostimato e sottoutilizzato a questi fini dai decisori pubblici, rispetto al loro reale potenziale costituito dalle 
possibilità di qualificazione strategica professionale del loro personale, della loro funzione di sistematizzazione tra i sempre crescenti flussi informativi, di intermediazione informativa, di acculturazione tecnologica nell'ambito delle TIC in relazione al digital divide generazionale, intergenerazionale, culturale, economico, e di preparazione alle molteplici esigenze della società della conoscenza in rete, infine, per lo storico ruolo e la significativa cultura di fiducia e prossimità sociale. È questo importante valore aggiunto che può fare di questa istituzione uno dei protagonisti di riferimento e avamposto nello sviluppo dell'intelligenza territoriale.

In una condizione generale, anche da noi delineata nei precedenti paragrafi come in questo caso da Corbineau (2005), dove spesso le azioni pubbliche per l'intelligenza territoriale sono relegate in una prospettiva eccessivamente semplificata di sviluppo economico e industriale, queste politiche possono invece acquisire una connotazione più ampia. L'intelligenza territoriale può così diventare lo strumento per uno sviluppo duraturo includendo le dimensioni gestionali-manageriali, le capacità di valutazione e analisi territoriale, il riconoscimento e lo sviluppo dei potenziali inespressi, nonché le capacità di gouvernance della dimensione sistemica, di quella strategica, della conduzione delle azioni e di quella socio-culturale (REIT). In questa prospettiva per Corbineau (2005) le mediateche oltre ad essere una risorsa socioculturale con la loro molteplice offerta di servizi sono anche e soprattutto uno spazio di interazioni sociali con la loro capacità di interfacciamento e di portale organizzato della conoscenza, ponendosi così come uno dei luoghi di eccellenza dove sviluppare e agire l'intelligenza territoriale come intelligenza collettiva nella prospettiva delineata da Pierre Lévy (1994).

Sono stati veramente molti gli studiosi di diversa provenienza geografica in ambito biblioteconomico e socio-umanistico che a partire dagli anni '60/'70 del secolo scorso hanno individuato nella biblio-mediateca di pubblica lettura quel "catalizzatore sociale» in grado di condensare, stimolare, raccordare, attivare, la creatività attraverso la conoscenza esperita attivamente nelle più diverse forme e modalità. Il loro numero e l'ampiezza delle loro considerazioni ed esperienze è tale che nelle dimensioni di questo saggio non possiamo inserirli tutti. Per un approfondimento in questo senso rimandiamo a due nostri precedenti lavori (Luciani, 2008 e 2009). Crediamo però che per una conferma ultima in relazione al rapporto tra il potenziale sviluppo e diffusione della creatività e l'istituto della biblio-mediateca pubblica sarebbe sufficiente riferirsi al Manifesto, redatto una prima volta nel 1972, e poi rivisto nel 1994, dall'International Federation of Library Association e adottato ufficialmente in entrambi i casi dall'UNESCO anche con queste parole: «La biblioteca pubblica, via di accesso locale alla conoscenza, costituisce una condizione essenziale per l'apprendimento permanente, l'indipendenza nelle 
decisioni, lo sviluppo culturale dell'individuo e dei gruppi sociali. Questo Manifesto dichiara la fede dell'UNESCO nella biblioteca pubblica come forza vitale per l'istruzione, la cultura e l'informazione e come agente indispensabile per promuovere la pace e il benessere spirituale delle menti di uomini e donne» (IFLA/UNESCO, 1994). Tutto il contenuto del Manifesto prosegue poi inquadrando con precisione il ruolo socio-culturale attivo di questo "centro informativo locale», mettendone in evidenza il significativo valore trasformativo in relazione alle persone e al territorio, e finendo col prendere direttamente in considerazione la dimensione della creatività affermando che tra i compiti della biblio-mediateca pubblica ci sono quelli di «offrire opportunità per lo sviluppo creativo della persona [e di] stimolare l'immaginazione e la creatività di ragazzi e giovani» (ibid.).

\section{DALle DiNAMiChe Di Flusso COMUNiCATIVO Digitale ALLE INTERAZIONI DI PROSSIMITÀ: AZIONI EDU-CREATIVE PER LA CITTÀ INTELLIGENTE}

Se i progetti collegati alla città e ai territori intelligenti sono spesso debitori nei confronti delle tecnologie della comunicazione che permettono l'accesso alla società connessa in rete, all'informazione e alla conoscenza, sviluppata attraverso tutte le dimensioni educative (formale, non formale, informale), allora la problematica relativa alle modalità/forme di accrescimento culturale attraverso la conoscenza, e quindi la capacità di trovare le risposte alle proprie domande conoscitive, dovrà essere affrontata e risolta soprattutto in funzione dello stimolo di quella creatività diffusa così necessaria all'intelligenza delle città. Questa è proprio, fin dai loro inizi, la funzione costitutiva delle biblio-mediateche di pubblica lettura insediate nelle città e nei territori: effettivi "portali di accesso alla conoscenza» gratuiti per la società tutta; spazi di incontro trasversale alle classi sociali grazie alla cultura come "comune denominatore»; luoghi di ricerca e di possibilità di selezione consapevole dei suoi esiti; ambienti di costruzione creativa mediale, sociale, e di conoscenza, collettiva e individuale, grazie alle risorse digitali off come online, ma anche attraverso altri mezzi analogici e all'incontro e al reciproco scambio consentito dalla prossimità in presenza; luoghi di vita e spazi pubblici capaci di suscitare fiducia nei loro utenti. Secondo Corbineau soprattutto quest'ultimo aspetto è "condition de l'intelligence (démocratique) en réseau» (2005).

Immersi, come siamo, in un costante flusso comunicativo digitale sociale e non, e come lo sono gli stessi istituti delle biblio-mediateche pubbliche, la dimensione concettuale e paradigmatica di riferimento per la distribuzione 
della conoscenza non è più soltanto il «deposito» ma soprattutto il «flusso» (Castells, 1996). In questa nuova dinamica comunicativa l'intermediazione delle informazioni e lo sviluppo della capacità di valutarle in relazione alle proprie finalità conoscitive resta fondante e fondamentale, sia per il loro utilizzo funzionale, quanto per la progressiva necessaria loro sistematizzazione in funzione di valore documentale e di memoria territoriale. Quello che cambia e che richiede un aggiornamento delle strutture e delle conoscenze/ competenze del personale preposto, sono i processi comunicativi, le tecnologie mediali e gli ambienti digitali in continua evoluzione oltre allo scarto conoscitivo, sempre esistito, ma anche in sempre maggiore espansione, tra gli specifici linguaggi mediali e la fruizione delle diverse forme comunicative mediali di riferimento.

L'intermediazione informativa dell'attualità in divenire è un compito e un ruolo che storicamente, lungo tutto il secolo scorso fino ad oggi, è stato affidato e in parte è stato acquisito sul campo dalle biblio-mediateche pubbliche e dai biblio-mediatecari che si occupano del funzionamento e dell'animazione di questi «centri informativi locali». Questa funzione era ed è oggi essenziale allo sviluppo individuale e collettivo. Anche grazie al coordinamento delle azioni educative non formali e informali che loro competono, i territori e le città possono mantenere vivo e vitale quel tessuto sociale composito, democraticamente creativo, che consente l'innesco e la necessaria adesione ai processi di cambiamento e di innovazione finalizzati alla dimensione in continuo divenire delle «città intelligenti». Quindi il biblio-mediatecario, aggiornato nelle sue conoscenze e competenze all'attuale contemporaneità comunicativa (Galliani, 1990 e 1993; Luciani, 2008), e inserito nel contesto di un istituto biblio-mediatecario pubblico evoluto e completo sia dal punto di vista tecnologico come dei servizi erogati (Luciani, 2008), risulta una figura chiave nel processo educativo relativo all' information literacy (Petrucco, 2003). Si tratta, come ci sembra evidente, di un ruolo fondamentale nell'attuale condizione socio-educativa di significativa sovrabbondanza e "confusione» informativa oltreché di problematicità costante e diffusa nell'attribuzione del valore di autorevolezza culturale. Questa istituzione, nel suo insieme di struttura e persone dedite alla sua animazione e organizzazione, svolge questo ruolo a diversi livelli e con diverse modalità. Essa si trova ad agire sia sul livello educativo informale, nel momento in cui attraverso un processo di selezione costruisce nel tempo i suoi cataloghi/archivi di contenuti, che ormai da qualche tempo devono riguardare anche sempre più quelli digitali off e online, quanto su quello formale nel momento in cui, in presenza e a distanza, attraverso la posta elettronica, i siti dinamici e interattivi, e i social software/network, si relaziona con l'utenza per svolgere il suo storico ruolo di orientamento, accompagnamento e supporto alla ricerca informativa. Inoltre, si colloca su 
quest' ultimo piano anche l'azione educativa possibile e strategica in funzione dell'accrescimento delle capacità di competizione di un territorio correlate allo sviluppo dell'intelligenza creativa delle persone, che si realizza attraverso la proposta formativa di veri e propri specifici corsi laboratoriali sull' infomation literacy (Petrucco, 2002) da offrire in modo trasversale a tutta la cittadinanza.

Le nuove tecnologie digitali della comunicazione multimediale interattiva, soprattutto portatili come gli smartphone e i tablet, unitamente allo sviluppo dei social software/network in Internet, consente oggi più di ogni altra epoca di immaginare e realizzare una consistente varietà di progetti di azione collaborativa ambientati nella città che mirano a sviluppare la dimensione della cittadinanza attiva e la riscoperta/conoscenza del territorio. Tra questi i più correlati al territorio, i più particolari e che maggiormente utilizzano le tecnologie della connessione in rete in mobilità (GPS, reti wireless, blutooth, RFID, GSM) sono i locative media. Sintetizzando una serie di definizioni desunte dalla rete, Giulia Bertone, in termini da lei ritenuti molto generali, li definisce come «un insieme di media location based che permettono di collegare i contenuti digitali ai luoghi geografici, valorizzando il carattere situato e geograficamente collocato dell'interazione» (2010, p. 196). Da questi progetti emerge in modo chiaro il ruolo di primo piano della localizzazione e il loro carattere di embodiment e quindi dell'importanza fondativa dell'esperienza situata e attiva in prima persona nei vari contesti di interazione. Sempre Giulia Bertone desumendolo da precedenti studi ne sintetizza così il senso: «Nella pratica e nella teoria, i locative media si affermano come nuovi strumenti creativi, in grado di dare vita ed esprimere relazioni significanti tra le persone, le comunità e i luoghi, collegando il materiale all'immateriale, gli oggetti ai contesti, i luoghi alle pratiche e agli usi» (ivi, p. 198). Da questi progetti di azione tecnologica mediale nei/per i territori e i suoi abitanti emerge chiaramente l'obiettivo del cambiamento sociale e della partecipazione creativa delle comunità, che è poi l'aspetto che abbiamo cercato di mettere maggiormente in luce in relazione alle dinamiche processuali, potenziali e non, dell'idea di smart city.

Dalla rassegna delle tematiche proposte dal City Centered Festival on locative media and urban community di San Francisco (Bertone, 2010), si può desumere che i progetti locative possono per esempio essere incentrati: sulla realizzazione individuale o collaborativa di mappe, che dando forma allo spazio urbano costruiscono identità; oppure attraverso la produzione di racconti digitali urbani favorendo la coesione sociale; o anche attraverso la delineazione di spazi urbani intelligenti, favorita dalla discussione su come l'informazione digitale off e online si espande nella città e su come la ridisegna; sull'accrescimento della propria consapevolezza in relazione agli altri abitanti, umani e non, che vivono la dimensione urbana; sulla scoperta della 
stratificazione storica della città nella dimensione integrata delle diverse storie locali (dell'architettura, dell'economia, dell'urbanistica, delle tradizioni, ecc.); sulla partecipazione attiva, situata e non neutrale, per co-immaginare e co-delineare degli approci agli spazi contestati e di conflitto nei contesti urbani; attraverso la dimensione ludica del gioco, strategica al fine di coinvolgere i giovani, con l'obiettivo di attivare interazioni tra differenti comunità anche in condizione di disagio e i quartieri in trasformazione. Come si può intuire, viste le tematiche affrontate e sviluppate da questa tipologia di progetti tecnologicamente imperniati e immersi nel paradigma esplorativoconoscitivo-creativo della contemporanea comunicazione digitale interattiva multimediale, potrebbero essere coordinati e proposti nell'ambito delle esperienze degli urban center più attivamente partecipativi. Nei casi più riusciti questi centri di «intelligenza creativa urbana» trovano sede proprio nelle biblio-mediateche delle città e ne diventano una parte correlata e attiva. Uno dei migliori esempi italiani in tal senso è quello della città di Bologna, che ha insediato il suo particolarmente attivo ed efficace urban center presso la "Biblioteca-Mediateca Sala Borsa», e che in effetti è anche una delle più complete e aggiornate esperienze mediatecarie italiane (Luciani, 2008). In questo modo l'istituto della mediateca pubblica, anche grazie e in virtù delle articolate sezioni di storia locale e della città sviluppate nel tempo, digitalizzate o meno che siano, che possono fungere da punto di partenza o da elementi di confronto/correlazione, assume la funzione di «centrale operativa» di progetti locative nella e per la città intelligentemente creativa.

Il concetto di educazione mediale (media education) e il suo relativo ambito disciplinare è progressivamente emerso ed è stato messo a punto nel tempo soprattutto nell'ambito degli studi dell'area delle scienze pedagogiche sui media a partire dagli anni '50/'60 del secolo scorso. Il sempre più imponente incremento della comunicazione mediale nella multi-dimensione della vita sociale delle persone, che è correlato alla costante maggiore diffusione delle tecnologie, da quelle analogiche a quelle attuali digitali, e al progressivo e costante aumento delle occasioni fruitive di tutti i testi mediali, ha messo in evidenza come sia impossibile relazionarsi consapevolmente ai processi politici ed economici, al rilievo/costruzione dell'identità personale e all'insieme dei processi educativi, senza tenere conto del ruolo socio-culturale dei media e senza arrivare a conoscerli il più consapevolmente possibile nella loro struttura ed azione (Galliani, 2009).

Leducazione mediale ha come obiettivo lo sviluppo sia della conoscenza critica quanto della partecipazione attiva. Gli obiettivi educativi sono quelli di sviluppare nelle persone in quanto fruitori dei media e dei loro prodotti testuali, soprattutto quelle più giovani - ma non esclusivamente -, delle consapevoli capacità interpretative, e allo stesso tempo di fornire quelle 
conoscenze e quelle competenze che gli permetteranno di diventare in prima persona produttori di testi mediali arrivando così a svolgere un effettivo ruolo di cittadinanza democraticamente attiva. Ponendo l'accento in modo particolare sui giovani, David Buckingham ne sintetizza così la correlazione: "Media education is about developing young people's critical and creative abilities» (2001). Proprio l'enfatizzazione dello sviluppo della creatività dei giovani e la loro diretta partecipazione attiva alla dimensione realizzativa permette ai processi di educazione mediale di arrivare a far sentire la loro voce ponendo le basi per delle future forme produttive di tipo comunicativo più democratiche ed inclusive. In relazione a quanto fin qui sostenuto relativamente alla prospettiva della relazione dinamica tra creatività e intelligenza e tra partecipazione attiva/creativa democratica e potenzialità di innovazione e resilienza, ci sembra che risulti evidente quanto questa azione formativa sociale sarebbe necessaria e strategica anche per le dinamiche realizzative di una città intelligentemente creativa, la smart city, che si sta affermando in gran parte proprio all'insegna dell'interconnessione digitale e quindi di fatto della mediazione comunicativa mediale.

Quest'ambito educativo perché possa avere effettivamente successo dovrebbe essere agito dal piano formale della scuola di ogni ordine e grado, da quella materna alle superiori, a quello informale delle istituzioni culturali e dell'associazionismo. Infatti, le esperienze più positive svolte in vari paesi hanno dimostrato che la migliore promozione e lo sviluppo dell'educazione mediale dipende dalla contemporanea presenza di una serie di fattori ideali tra loro interdipendenti e dall'alleanza e collaborazione tra diverse strutture sociali educative ugualmente interessate: il tutto distribuito in parte sul piano internazionale, in parte su quello nazionale e in parte su quello locale (Buckingham, 2001). Sul piano locale, oltre alla scuola e allo stesso tempo anche in collaborazione con questa, l'istituto della mediateca pubblica diventa allora necessariamente uno dei luoghi privilegiati - diremmo centrale - dove agire l'educazione mediale nelle sue molteplici possibili modalità didattiche e proposte formative, in relazione a tutti i possibili media comprendendo anche la comunicazione digitale online interattiva del web 2.0, e anche per quanto riguarda i diversi momenti realizzativi, casomai adottando come tematiche proprio quelle, numerossissime, offerte dalla stessa città.

La stupefacente accelerazione ed espansione informativa innescata dai flussi comunicativi digitali in rete e non, sempre più progressivamente e significativamente co-distribuita e co-costruita in modo partecipato (Jenkins, 2006), ci permette nuove imponenti potenzialità conoscitive, generando però al contempo significative modificazioni della dimensione cognitiva e dei suoi processi (McLuhan, 1964; Ong, 1982). Questa condizione fattuale così dinamica e instabile, che per alcuni è ormai diventata sistemica, finisce per 
ingenerare divari di conoscenza e spesso rapide obsolescenze nell'operatività a questa correlata (acquisizione, gestione), sia a livello di pratiche sociali di cittadinanza attiva e di acculturazione, quanto a livello lavorativo. $\grave{E}$ anche in relazione a questa problematica e alle sue conseguenze che ormai da tempo le politiche socio-educative delle nazioni più progredite sono improntate alla prospettiva formativa del lifelong, lifewide, lifedeep learning (Commissione Europea, 1995; UNESCO, 1997).

In questo contemporaneo contesto socio-culturale di comunicazione e di necessità di apprendimento continuo, nonché di sistematizzazione/aggiornamento della conoscenza acquisita, spicca una proposta esemplificativa che ha di recente (settembre 2013) raggiunto il suo compimento realizzativo e ha iniziato la sua azione operativa socio-educativa: la Library of Birmingham (LoB) (Blewitt \& Gambles, 2010). Si tratta di una biblioteca pubblica multimediale (mediateca) immaginata e progettata proprio con l'obiettivo di fornire un nuovo spazio dove porre al centro l'apprendimento per tutta la vita e lo sviluppo della conoscenza. Le modalità operative che sono state concepite per conseguire questo risultato sono quelle di implementare in modo importante e dinamico delle collaborazioni con diversi enti / società private, con il pubblico, con gli organismi del terzo settore, così come anche con i singoli cittadini. L'idea complessiva è quella di un organismo in continua evoluzione e sviluppo al fine di meglio raccordarsi al progressivo divenire della contemporaneità, articolandosi in un costante processo di funzionale crescita culturale e di adeguamento alle emergenze formative della società come quelle derivanti dall'affermazione della cultura digitale. Ad ulteriore conferma di quanto da noi sostenuto nell'articolazione di questo nostro saggio, le consegne chiave ricevute dai decisori politici e le linee guida centrali sono per l'appunto: arte e creatività, cittadinanza attiva e comunità, impresa e innovazione, l'apprendimento con una particolare attenzione alle nuove competenze, e infine la nuova ecologia dei media, concetto che crediamo si possa porre alla base delle azioni educative correlate alla digital literacy. Questa mediateca si pone come punto di riferimento centrale nel cuore del quartiere culturale della città a partire dal suo design, e non solo da un punto di vista architettonico, ma anche nel senso del progetto edu-formativo che rappresenta. La LoB persegue con piena consapevolezza socio-culturale l'obiettivo di stimolare la crescita economica sostenibile, la riqualificazione urbana e l'inclusione sociale, offrendo una vasta gamma di nuovi servizi correlati all'apprendimento digitale, spazi di comunità in presenza e online, oltre a nuove occasioni per l'utilizzo e la fruizione delle sue significative collezioni di archivi documentari, di fotografia, di immagini in movimento, e di rari libri. Infine, la LoB offre nuovi spazi fisici agibili dai creativi per le loro finalità progettuali e realizzative, per l'azione e l'organizzazione legata alla dimensio- 
ne culturale, per nuove imprese, e per lo sviluppo della conoscenza in senso ampio nella dimensione non formale e informale.

In diverse città di vari paesi del mondo si stanno affermando tutta una serie di nuovi servizi e azioni sociali innovative immaginate e realizzate all'insegna della delineazione delle nuove possibili forme degli spazi pubblici e dell'apertura alla comunità col fine di sviluppare la collaborazione, la conoscenza e la creatività. Contemporaneamente e significativamente svincolate dalla relazione diretta con le amministrazioni e le varie istituzioni pubbliche compaiono anche sempre di più delle iniziative di condivisione della conoscenza proposte "dal basso", direttamente dai cittadini, che danno senso e valore al concetto di «cittadinanza attiva».

Di seguito a titolo esemplificativo ne indichiamo qualcuna a partire da uno studio canadese relativo alla città di Montréal (Audette-Chapdelaine, 2011): azioni locali e collettivamente partecipative come un particolare tipo di «laboratorio mediale vivente» (Mandalab, ENOLL, Living Labs) che si occupa di digital divide generazionale come anche più recentemente di tecniche sociali e di cittadinanza partecipata preparando e mettendo in collegamento i cittadini, le imprese e i ricercatori attorno ad un progetto socialmente orientato; attivazione di spazi di coworking che permette alle persone già in grado di svolgere telematicamente il proprio lavoro anche da casa di soddisfare anche la necessità vitale di socializzazione solidale (niente a che vedere con l'aspetto di un centro d'affari, ma piuttosto uno spazio di scambio e compartecipazione dove le persone sono immerse in un ambiente amicale, stimolante e a "creatività diffusa»); attivazione di spazi creativi di costruzione digitale di nuovi oggetti. Il fab lab è un'esperienza derivata dal MIT e si concretizza come un laboratorio di costruzione, di apprendimento, di aiuto reciproco e mutualità, attivamente partecipato che offre alle persone tutta una serie di strumenti e tecnologie digitali ad uso gratuito come per esempio una stampante 3D. Nel caso fosse necessario le attività possono essere svolte con la supervisione di un responsabile esperto del laboratorio. I partecipanti condividono poi la documentazione dei loro progetti/realizzazioni attraverso la rete in spazi online a libero accesso e completamente disponibili per la condivisione e l'eventuale miglioramento, favorendo in questo modo anche la co-progettazione a distanza tra gruppi.

Anche in questi ultimi casi, come negli altri da noi precedentemente presentati, risulta abbastanza conseguente come l'istituto della biblio-mediateca pubblica si potrebbe confermare come lo spazio strategico nella città e per la città, ospitando queste e altre iniziative con la stessa cifra stilistica partecipativa e collaborativa in presenza, ma comunque sempre pienamente inseriti nella cultura digitale contemporanea e futuribile, rafforzando ancora di più la funzione di stimolatore creativo delle «intelligenze mediali». 


\section{CONCLUSIONI}

Se, come ci dice Charles Landry, oggi la creatività di una città viene di solito esclusivamente considerata in relazione alla sua scena artistica e culturale piuttosto che rispetto alle capacità che dimostra in campo scientifico, ingegneristico, tecnologico o anche per quelle appartententi ad altri campi di attività, e se la scena culturale, soprattutto da un punto di vista mediatico, appare più seducente di altre, allora una compensazione attiva sul campo può derivare proprio dai «musei e [dal]le infrastrutture educative [che] possono contribuire nel generare futura capacità di innovazione» (2009, p. 476). Tra queste, come ci sembra di avere dimostrato con il nostro discorso argomentativo, la mediateca pubblica potrebbe acquisire una funzione socio-culturale particolarmente attiva e funzionale relativamente ai processi di attivazione della "creatività diffusa» nella città e nei territori, oggi anche chiaramente sempre di più all'insegna della dimensione digitale e della digital literacy, come necessaria premessa e costante accompagnamento allo sviluppo della smart city.

\section{RIFERIMENTI BIBLIOGRAFICI}

Agnoli, A. (2009). Le piazze del sapere. Biblioteche e libertà. Roma - Bari: Laterza.

Audette-Chapdelaine, V. (2011). Éspaces physiques et pratiques émergentes - Comment les bibliothèques publiques peuvent y participer. Bulletin des Bibliothèques de France, 6. http://bbf.enssib.fr/consulter/bbf-2011-06-0034-003.

Bertone, G. (2010). Locative media: una perlustrazione al City Centered Festival. REM - Ricerche su Educazione e Media, 2(2), 193-220.

Blewitt, J., \& Gambles, B. (2010). The Library of Birmingham project: Lifelong learning for the digital age. Journal of Adult and Continuing Education, 16(2), 52-66.

Buckingham, D. (2001). Media education. A global strategy for development. Policy paper prepared for UNESCO. http://portal.unesco.org/ci/en/ev.php-URL ID $=5681 \&$ URL_DO=DO_TOPIC\&URL_SECTION=201.html.

Castells, M. (1996). The rise of the network society. The information age: Economy, society and culture, Vol. 1. Oxford: Blackwell.

Commissione Europea (1995). Libro bianco su istruzione e formazione - Insegnare e apprendere - Verso la società conoscitiva. http://ec.europa.eu/white-papers/ index_it.htm.

Commissione Europea (2009). Manifesto per la creatività e l'innovazione in Europa. http://www.create2009-italia.it/content/index.php?action=read_pagina\&id_ cnt $=8443$. 
Commissione Europea (2010). Libro verde - Le industrie culturali e creative, un potenziale da sfruttare. http://ec.europa.eu/green-papers/index_it.htm.

Consiglio d'Europa (2008). Libro bianco sul dialogo interculturale - Vivere insieme in pari dignità. http://ec.europa.eu/green-papers/index_it.htm.

Corbineau, B. (2005). Intelligence territoriale et societe en reseau. Les mediatheques de proximite, portails de la connaissance. In 4 e Tic \& Territoire: quels développements?, Journée sur les systèmes d'information élaborée, île Rousse. http:// isdm.univ-tln.fr/PDF/isdm22/isdm22_corbineau.pdf.

Dewey, J. (1917). The need for a recovery of philosophy. In AA.VV., Creative intelligence. Essays in the pragmatic attitude. New York: Henry Holt \& Co. http:// www.gutenberg.org/files/33727/33727-h/33727-h.htm\#Page_3.

Dewey, J. (2012). Democrazia e educazione (trad. it.). Milano: Sansoni (ed. orig., Democracy and education. NewYork: The Macmillan Company, 1916).

Florida, R. (2003). L'ascesa della nuova classe creativa. Stile di vita, valori e professioni (trad. it.). Milano: Mondadori (ed. orig., The rise of the creative class: And how it's transforming work, leisure and everyday life. New York: Basic Books, 2002).

Galliani, L. (1983). Ricerca-intervento nel territorio e ruolo degli audiovisivi. Quaderni di Comunicazione Audiovisiva, 2(1), Aprile, 54-65.

Galliani, L. (1990). Les nouvelles technologies et les nouveaux langages dans le développement de la ville éducatrice. In Morel \& Jordi, 1990.

Galliani, L. (1993). Nuove tecnologie e nuove professioni nella comunicazione multimediale. Sociologia del Lavoro, 49, 74-81.

Galliani, L. (2000). Didattica come tecnologia. In AA.VV., Pedagogia Didattica Tecnologia. Pisa - Roma: Istituti Editoriali e Poligrafici.

Galliani, L. (2009). La ricerca sull'educazione mediale. REM - Ricerche su Educazione e Media, 1(1), 9-23.

Gardner, H. (1991). Aprire le menti. La creatività e i dilemmi dell'educazione (trad. it.). Milano: Feltrinelli (ed. orig., To open minds. Chinese clues to the dilemma of contemporary education. New York: Basic Books, 1989).

Gardner, H. (2007). Cinque chiavi per il futuro (trad. it.). Milano: Feltrinelli (ed. orig., Five minds for the future. Boston: Harvard Business School Press, 2006).

Gatti, R. (2012). Smart cities learning: la tecnologia per una città partecipata e formativa. Intervista a Carlo Giovannella. http://smartinnovation.forumpa.it/story/69595/ smart-cities-learning-la-tecnologia-una-citta-partecipata-e-formativa.

Giovannella, C., \& Baraniello, V. (2012). Smart city learning. International Journal of Digital Literacy and Digital Competence, 3(4), 1-15.

Giovannella, C., Iosue, A., Tancredi, A., Cicola, F., Camusi, A., Moggio, F., Baraniello, V., Carcone, S., Coco, S. (2013). Scenarios for active learning in smart territories. Interaction Design and Architecture(s) Journal, 16, 7-16. http:// www.mifav.uniroma2.it/inevent/events/idea2010/doc/16_1.pdf. 
IFLA/UNESCO (1994). Public Library Manifesto. http://archive.ifla.org/VII/s8/ unesco/ital.htm.

Il Sole 24 Ore (2012). Manifesto della Cultura. http://www.statigeneralidellacultura. ilsole24ore.com/allegati/ManifestoDellaCultura.pdf.

ISTAT (2012). Cittadini e nuove tecnologie. http://www.istat.it/it/archivio/78166.

Jenkins, H. (2006). Convergence culture: Where old and new media collide. New York: University Press (trad. it., Cultura convergente. Milano: Apogeo, 2007).

Landry, C. (1998). Helsinki: Towards a creative city. Seizing the opportunity and maximizing potential. Helsinki: City of Helsinki.

Landry, C. (2009). City making. L'arte di fare la città (trad. it.). Torino: Codice (ed. orig., The art of city making. London - Sterling, VA: Earthscan, 2006).

Levy, P. (1994). Lintelligence collective. Pour une anthropologie du cyberspace. Paris: La Découverte (trad. it., Lintelligenza collettiva. Per un'antropologia del cyberspazio. Milano: Giangiacomo Feltrinelli, 1996).

Luciani, L. (2008). Dalla Biblioteca alla Mediateca. Scenari di educazione ai media e un progetto territoriale. Tesi di dottorato in Scienze pedagogiche e didattiche, Università degli Studi di Padova. http://paduaresearch.cab.unipd.it/945/.

Luciani, L. (2009). Dalla mediateca didattica alla biblio-mediateca: alle radici di un percorso comune di media education. Giornale Italiano della Ricerca Educativa, 2(3), Dicembre, 105-120.

McLuhan, M. (1964). Understanding media: The extensions of man. New York: McGrawHill (trad. it., Gli strumenti del comunicare. Milano: NET - Nuove Edizioni Tascabili, 2002).

Menduni, G. (2012). La città educante. http://smartinnovation.forumpa.it/story/69772/ la-citta-educante.

MIUR (2012a). Avviso per la presentazione di idee progettuali per smart cities and communities and social innovation. http://attiministeriali.miur.it/anno-2012/ luglio/dd-05072012.aspx.

MIUR (2012b). Graduatorie finali dei Progetti finanziati nell'ambito dell'Avviso D.D. 84/Ric. http://attiministeriali.miur.it/anno-2012/settembre/dd-28092012.aspx.

Morel, S., \& Jordi, F. (Eds.). (1990). La Ciudad Educatora - La Ville Éducatrice, Barcelona: Regidoria d'Edicions i Publicacions, Ajuntament de Barcelona.

Ong, J. W. (1982). Orality and literacy. The technologizing of the word. London - New York: Methuen (trad. it., Oralità e scrittura. Le tecnologie della parola. Bologna: il Mulino, 1986).

Petrucco, C. (2002). Laboratorio di ricerca delle informazioni in Internet per la didattica. Studium Educationis. Rivista per la Formazione nelle Professioni Educative, Numero speciale: Educazione e Media, Coordinamento di L. Galliani e R. Maragliano, 3, 735-746.

Petrucco, C. (2003). Ricercare in rete. Lecce: Pensa MultiMedia. 
Pew Internet (2012). Demographics of Internet users. http://pewinternet.org/TrendData-\%28Adults\%29/Whos-Online.aspx.

Riva, M. (2010). Nuvole e crisi. Nuove frontiere per le industrie creative. $R E M-R i$ cerche su Educazione e Media, 2(2), 261-274.

Rullani, E. (2006). Economia della conoscenza. Creatività e valore nel capitalismo delle reti. Roma: Carocci.

UNESCO (1997). Dichiarazione finale. Quinta Conferenza Internazionale sull'educazione degli adulti. http://www.edscuola.it/archivio/norme/varie/amburgo 1997. pdf.

Yvon, T. (2010). Alcune osservazioni preliminari sul Libro verde dell'Unione Europea - Liberare le potenzialità delle industrie culturali e creative. REM-Ricerche su Educazione e Media», 2(2), 275-284.

\section{SITOGRAFIA}

Creative Cities Network, http://www.unesco.org/new/en/culture/themes/creativity/ creative-cities-network/.

ENOLL - European Network of Living Lab, http://www.openlivinglabs.eu/.

REIT - Territorial Intelligence Portal. Knowledge, methods, governance and tools for action, http://www.intelligence-territoriale.eu/.

\section{VIDEOGRAFIA}

Landry, C. (2012). Creative cities vs Smart cities. Il ruolo delle tecnologie. Keynote tenuto a Bologna nel corso di Smart City Exhibition 2012. https://www. youtube.com/watch?v=ImHJPz1ggpw\&list=PLJqDgROEqMMWwdpj15PaoxBSsEYY0D70\&index $=1$.

Landry, C. (2013). Città creative. Sintonizzare il contemporaneo con il futuro. Intervista di A. Granelli nell'ambito dei cicli di Conferenze Segnavie. Orientarsi nel mondo che cambia. Fondazione Cariparo. http://www.youtube.com/watch?v=85GF_f2CtU\&list=UUI1wL3JG34uXquGkGgQg6kQ\&feature=c4-overview.

\section{Riassunto}

A fronte del diffondersi sempre maggiore sul piano comunicativo dell'insieme terminologico di «smart city» si riscontra una notevole polisemia del senso a questo attribuito e del suo significato d'uso. Come una moda sempre più diffusa e fin troppo facile da adottare, la 
"smart city" viene sempre più proclamata, proposta, e indicata come traguardo dai decisori politici. Molti ambiti applicativi e azioni amministrative sono denominate "smart», intelligenti. Ma cos'è in realtà una città intelligente? Quando possiamo considerarla effettivamente tale? Qual è il rapporto tra l' «intelligenza» dei sistemi organizzativo-tecnologici della vita sociale, dell'ambientelterritorio, e l'intelligenza delle persone che vivono la città? Sullo sfondo dello sviluppolevoluzione delle città c'è un rapporto tra l'intelligenza e la creatività? Attraverso le risposte a queste domande, risultato di una disamina teorica logicoconsequenziale, questo contributo si pone l'obiettivo di proporre e sostenere il valore connesso, in relazione alla diffusionelacquisizione critica della cultura digitale, alla compiuta realizzazione di un capillare e diffuso sistema di mediateche proposto come uno dei cardini per l'effettiva realizzazione delle città intelligenti. Si tratta di un'azione che non dovrebbe essere oltremodo derogabile in Italia, che nei confronti del panorama attuale degli altri paesi europei e di quelli economicamente più avanzati, ne ha anche maggiore necessità in quanto è significativamente deficitaria rispetto a questa specifica tipologia di strutture, del loro relativo irradiamento culturale, e dello sviluppo socio-creativo umano di «intelligenza digitale» di cui sono potenzialmente portatrici.

Parole chiave: Città intelligente, Creatività, Cultura digitale, Educazione mediale, Mediateca. 